\title{
Los Conocimientos de Enfermería como Instrumento para el Fomento de la Diálisis Peritoneal
}

\author{
Jesús Lucas Martín Espejo - Francisco Cirera Segura
}

Enfermeros

Unidad de Gestión Clínica Uro.Nefrológica. H.H.U.U. Virgen del Rocío. Sevilla.

\section{Resumen}

Si bien está probado que la diálisis peritoneal es una opción de tratamiento de la Enfermedad Renal Crónica Terminal al igual que la hemodiálisis, su uso es menor.

Se ha debatido mucho sobre las posibles causas para su escaso desarrollo en España, apuntándose que la falta de impulso de la diálisis peritoneal puede deberse a que tanto médicos como Enfermería, no tengan la suficiente información o formación sobre esta opción de tratamiento.

Como objetivo nos propusimos identificar el grado de conocimiento sobre la diálisis peritoneal que tiene el personal de Enfermería, con el fin de tomar las medidas necesarias para el fomento y la buena práctica de este tratamiento.

La muestra estaba constituida por un total de 66 enfermeros.

Para identificar el grado de conocimiento de Enfermería sobre la diálisis peritoneal, se diseñó un cuestionario.

Correspondencia:

Jesús Lucas Martín Espejo

C/ Gólgota 16, $2^{\circ}$

41007. Sevilla

Email: jlucasmartin@hotmail.com
Como resultado obtuvimos que sólo el 57,6\% sabía realizar un cambio de bolsa y un 30,3\% reconocieron manejar una cicladora.

Más del 86,4\% sabían reconocer los síntomas de infección de la inserción y la infección peritoneal, pero un $55 \%$ no sabría actuar ante las complicaciones más frecuentes en diálisis peritoneal.

Hemos podido comprobar que el grado de conocimiento general sobre la diálisis peritoneal es limitado. Pensamos que esto puede influir en el fomento y la buena práctica de la diálisis peritoneal, a pesar que el 69,2\% piensan que los resultados de esta modalidad de tratamiento para un paciente estándar son iguales o mejores que con la hemodiálisis.

PALABRAS CLAVE:

- CONOCIMIENTOS

- INSTRUMENTO

- DIÁLISIS PERITONEAL

- FORMACIÓN

\section{Nursing knowledge as an instrument for the promotion of peritoneal dialysis}

\section{Abstract}

Although it is proven that peritoneal dialysis, like haemodialysis, is an option for treating Terminal Chronic Renal Disease, it is used less. 
There has been considerable debate on the possible causes of its limited development in Spain, and it has been suggested that the lack of promotion of peritoneal dialysis may be due to the fact that both doctors and nurses have insufficient information or training on this treatment option.

We set ourselves the aim of identifying the degree of knowledge that Nursing staff have of peritoneal dialysis, in order to take the necessary measures to promote this treatment and good practice.

The sample was made up of a total of 66 nurses.

To identify Nursing staff's degree of knowledge of peritoneal dialysis, a questionnaire was designed.

As a result we found that only $57.6 \%$ knew how to change a bag and $30.3 \%$ recognized how to handle a cycler.

More than $86.4 \%$ knew how to recognize the symptoms of infection at the insertion point and peritoneal infection, but 55\% would not know how to act in response to the most frequent complications in peritoneal dialysis.

We have found that the general degree of knowledge of peritoneal dialysis is limited. We think that this can influence the promotion of and good practice in peritoneal dialysis, although $69.2 \%$ think that the results of this type of treatment for a standard patient are equal to or better than with haemodialysis.

\section{KEY WORDS:}
- KNOWLEDGE
- INSTRUMENT
- PERITONEAL DIALYSIS
- TRAINING

\section{Introducción}

Si bien está probado que la Diálisis Peritoneal (D.P.) es una opción de tratamiento de la Enfermedad Renal Crónica Terminal (E.R.C.T.) al igual que la
Hemodiálisis (H.D.) $)^{1}$ la realidad es que su uso es mucho menor en la mayoría de los centros de nuestro país ${ }^{2}$. Más, si tenemos en cuenta el importante consenso que existe sobre el denominado PD first, o D.P. como mejor opción para el inicio del tratamiento sustitutivo ${ }^{1}$.

Se ha debatido mucho sobre las posibles causas para el escaso desarrollo de la D.P. en España, y un estudio Europe ${ }^{3}$ que asigna niveles de causalidad, consideraba entre ellas la escasez de recursos materiales y personales, la falta de infraestructura hospitalaria, la falta de entrenamiento y experiencias limitadas y la insuficiente información recibida por los pacientes sobre la D.P. Añadiríamos a estas causas los criterios económicos e incluso en algunas autonomías, criterios políticos. En definitiva son múltiples factores los que han determinado que no se implante o no evolucione esta modalidad de tratamiento en algunos centros. Incluso en aquellos que cuentan con medios y poblaciones similares, la D.P. no alcanza una implantación igualitaria debido posiblemente, a que en las consultas de Enfermedad Renal Crónica Avanzada no se informa con el mismo énfasis en ambas técnicas (ya que cuando se presenta la información de manera ecuánime, sin sesgos, aproximadamente la mitad de los pacientes optan por la D.P.) ${ }^{4,5,6}$, a que los pacientes que ingresan por urgencias son dirigidos a H.D. y suelen desconocer la existencia de otros tratamientos ${ }^{5}$ y al uso de la D.P. como último recurso en pacientes con falta de acceso $\operatorname{vascular}^{7-8}$, o de poblaciones extremas ${ }^{8}$.

Una de las razones apuntada en todos los foros de debates y aun no suficientemente demostrada, es si la falta de impulso de la D.P. puede deberse a que el personal sanitario, tanto médicos como Enfermería, no tengan la suficiente información o formación sobre esta opción de tratamiento ${ }^{4-9}$.

Es cierto que las especialidades en Medicina están generando hoy sub-especialidades que hacen que muchas veces no podamos abarcar todo aquello (técnicas, tratamientos, pruebas, etc.) que conlleva nuestro campo de trabajo, pero sí creemos importante poseer al menos los conocimientos básicos para poder actuar en determinadas circunstancias e informar adecuadamente a nuestros pacientes, que además tienen este derecho adquirido por ley ${ }^{10}$.

En este trabajo nos planteamos como objetivo identificar el grado de conocimiento sobre la D.P. que tiene el 
personal de Enfermería, con el fin de tomar las medidas necesarias para el fomento y la buena práctica de esta modalidad de tratamiento.

\section{Material y métodos}

La muestra estaba constituida por todo el personal de Enfermería de nuestro Servicio $(n=57)$, aunque durante el periodo de recogida de cuestionarios, se solicitó al personal eventual con más de un año de trabajo en el servicio de Nefrología $(n=9)$ que la completara, siendo la muestra total 66 enfermeros.

Para identificar el grado de conocimiento que tiene Enfermería sobre la D.P. en nuestro Servicio, se diseñó un cuestionario. En ningún momento pensamos realizar una evaluación del personal, sino identificar los puntos donde sería necesaria una intervención para el fomento y la formación del personal en D.P. Tanto la participación en el estudio como la cumplimentación del cuestionario, fue anónima y voluntaria.

El cuestionario se dividió en 3 apartados. El primero evaluaba la formación individual en D.P. Un segundo apartado, dedicado a los conocimientos para la realización del tratamiento y el tercero, estaba dirigido específicamente al personal de Enfermería que realiza D.P. En total, la encuesta estaba constituida por 25 preguntas cerradas (55 ítems) y una abierta donde se pudiera añadir cualquier observación o sugerencia por parte de Enfermería. Las preguntas se redactaron en base a la bibliografía existente para la formación de pacientes en D.P. ${ }^{11,12,13,14,15,16,17,18}$.

El tratamiento estadístico se realizó con el programa S.P.S.S. 17.0, realizando tablas de distribución de frecuencias y porcentajes. Por último, se interpretaron los resultados obtenidos, y comentado los hallazgos.

\section{Resultados}

La muestra estaba compuesta por 66 enfermeros/as del Servicio de Nefrología de nuestro hospital. El tiempo de experiencia profesional de los mismos fue de 20,02 $+10,16$ años, y dentro del servicio de Nefrología 15,2 $+11,1$ años. La distribución por unidades dentro del servicio se muestra en la Tabla 1.

\begin{tabular}{l|c|}
\hline Servicio & Número de Profesionales \\
\hline Hemodiálisis & $n=38(57,6 \%)$ \\
\hline Trasplante Renal & $n=12(18,2 \%)$ \\
\hline Hospitalización & $n=11(16,7 \%)$ \\
\hline Diálisis Peritoneal & $n=5(7,5 \%)$ \\
\hline
\end{tabular}

Tabla 1. Distribución de los profesionales por Servicio.

Se preguntó por las opciones de tratamiento de la E.R.C.T. que conocían y practicaban. Hemos desglosado los resultados por servicio ya que el personal de trasplante renal en nuestra unidad, atiende también la unidad hospitalaria de D.P. El $100 \%$ del personal de trasplante renal $(n=12)$ y D.P. $(n=5)$ conocía las tres modalidades de tratamiento y realizaba las dos técnica de depuración extrarrenal. Los resultados del personal de H.D. y hospitalización se muestran en la Tabla 2.

En general, el 54,5\% ( $n=36)$ de los encuestados consideraba que para un paciente estándar, ambas técnicas

\begin{tabular}{|c|c|c|c|c|c|c|}
\hline \multirow{2}{*}{\multicolumn{2}{|c|}{ SERVICIO }} & \multicolumn{2}{|c|}{ HEMODIÁLISIS } & \multicolumn{3}{|c|}{ HOSPITALIZACIÓN } \\
\hline & & \multirow{2}{*}{$\begin{array}{c}\text { SÍ } \\
100 \%(n=38)\end{array}$} & \multirow{2}{*}{ NO } & \multirow{2}{*}{$\begin{array}{c}\text { SÍ } \\
90,9 \%(n=10)\end{array}$} & \multirow{2}{*}{$\begin{array}{l}\text { NO } \\
-\end{array}$} & \multirow{2}{*}{$\begin{array}{c}\text { NO CONTESTA } \\
9,1 \%(n=1)\end{array}$} \\
\hline \multirow{3}{*}{ 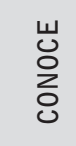 } & Hemodiálisis & & & & & \\
\hline & Diálisis Peritoneal & $78,9 \%(n=30)$ & $21,1 \%(n=8)$ & $90,9 \%(n=10)$ & - & $9,1 \%(n=1)$ \\
\hline & Trasplante Renal & $71,1 \%(n=27)$ & $28,9 \%(n=11)$ & $90,9 \%(n=10)$ & - & $9,1 \%(n=1)$ \\
\hline \multirow{2}{*}{ 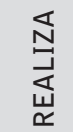 } & Hemodiálisis & $100 \%(n=38)$ & - & $27,3 \%(n=3)$ & $18,2 \%(n=2)$ & $54,5 \%(n=6)$ \\
\hline & Diálisis Peritoneal & $26,3 \%(n=10)$ & $73,7 \%(n=28)$ & $36,4 \%(n=4)$ & $9,1 \%(n=1)$ & $54,5 \%(n=6)$ \\
\hline
\end{tabular}

Tabla 2. Conocimientos y práctica de las modalidades de tratamiento de la Enfermedad Renal Crónica Terminal en los servicios de hemodiálisis y hospitalización. 
de depuración extrarrenal son igual de válidas. EI $16,7 \%(n=11)$ pensaban que la H.D. es mejor técnica, el $13,6 \%(n=9)$ pensaban que es mejor la D.P. y el $15,2 \%(n=10)$ no contestó esta pregunta.

En lo concerniente a la formación específica en D.P, el 57,6\% ( $n=38)$ de la Enfermería del Servicio había recibido algún tipo formación. El 31,6\% $(n=12)$ de ellos no había realizado ningún curso teórico sobre D.P. y el $86,8 \%(n=33)$ había recibido formación práctica sobre la misma.

El $46,9 \%$ de los encuestados $(n=31)$ ha trabajado más de un año en alguna unidad de nuestro servicio donde se atiende a los pacientes de D.P. pero sólo el $9,1 \%(n=6)$ ha realizado el entrenamiento de algún paciente en D.P.

La información que el personal de nuestro Servicio tenía sobre el programa de D.P, y los medios y actividades que este realiza, se evaluaron mediante 4 preguntas. El 95,5\% ( $n=63)$ conocía la existencia de la unidad de D.P, el $89,4 \%(n=59)$ el programa de D.P, el $95,5 \%$ $(n=63)$ identificaba un personal específico destinado a este programa y el $27,2 \%(n=18)$ pensaba que existía un programa de formación para el personal sanitario en esta modalidad de tratamiento, cuando no es así.

El $89,4 \%(n=59)$ de los encuestados identificaba los síntomas de la infección de la inserción del catéter peritoneal, aunque sólo el 13,6\% ( $n=9)$ conocía la clasificación que hace Twardowski de los mismos. El $86,4 \%(n=57)$ conocía los síntomas de la infección peritoneal y el $7,6 \%(n=5)$ la clasificación de permeabilidad del peritoneo según Twardowski.

Los resultados sobre las actuaciones ante las complicaciones habituales en D.P. en base al protocolo del servicio, se muestran en la Tabla 3 y sobre la realización de la técnica, en la Tabla 4.

\begin{tabular}{|c|c|c|c|}
\hline Sabría qué hacer en caso de: & SÍ & NO & NO CONTESTA \\
\hline Hemoperitoneo. & $22,7 \%(n=15)$ & $74,3 \%(n=49)$ & $3 \%(n=2)$ \\
\hline Infección peritoneal. & $45,5 \%(n=30)$ & $54,5 \%(n=36)$ & - \\
\hline Rotura de catéter. & $30,3 \%(n=20)$ & $69,7 \%(n=46)$ & - \\
\hline Fuga por la inserción. & $24,3 \%(n=16)$ & $72,7 \%(n=48)$ & $3 \%(n=2)$ \\
\hline No infusión de la bolsa. & $33,3 \%(n=22)$ & $66,7 \%(n=44)$ & - \\
\hline No drenaje del líquido peritoneal. & $36,4 \%(n=24)$ & $63,6 \%(n=42)$ & - \\
\hline Contaminación del prolongador. & $36,4 \%(n=24)$ & $63,6 \%(n=42)$ & - \\
\hline Rotura o desconexión del prolongador. & $34,8 \%(n=23)$ & $65,2 \%(n=43)$ & - \\
\hline
\end{tabular}

Tabla 3. Actuaciones de Enfermería ante las complicaciones habituales en Diálisis Peritoneal.

\begin{tabular}{|l|l|c|c|}
\hline Sabría realizar: & \multicolumn{1}{|c|}{ SÍ } & NO & NO CONTESTA \\
\hline Cambio de bolsa. & $57,6 \%(n=38)$ & $39,4 \%(n=26)$ & $3 \%(n=2)$ \\
\hline Cambio de prolongador. & $36,4 \%(n=24)$ & $59,1 \%(n=39)$ & $4,5 \%(n=3)$ \\
\hline Programar la cicladora. & $34,8 \%(n=23)$ & $60,7 \%(n=40)$ & $4,5 \%(n=3)$ \\
\hline Montar la cicladora. & $33,3 \%(n=22)$ & $62,2 \%(n=41)$ & $4,5 \%(n=3)$ \\
\hline Desconexión de la cicladora. & $36,4 \%(n=24)$ & $59,1 \%(n=39)$ & $4,5 \%(n=3)$ \\
\hline Solucionar las alarmas de la cicladora. & $30,3 \%(n=20)$ & $63,7 \%(n=42)$ & $6 \%(n=4)$ \\
\hline Kt/V en Diálisis Peritoneal. & $16,7 \%(n=11)$ & $78,8 \%(n=52)$ & $4,5 \%(n=3)$ \\
\hline Test de equilibrio peritoneal. & $12,1 \%(n=8)$ & $80,3 \%(n=53)$ & $7,6 \%(n=5)$ \\
\hline Sabría cumplimentar hoja de balances en: & $\mathbf{S I ́}$ & $\mathbf{N 0}$ & NO CONTESTA \\
\hline Diálisis Peritoneal Continua Ambulatoria. & $48,5 \%(n=32)$ & $48,5 \%(n=32)$ & $3 \%(n=2)$ \\
\hline Diálisis Peritoneal Automatizada. & $34,8 \%(n=23)$ & $62,2 \%(n=41)$ & $3 \%(n=2)$ \\
\hline
\end{tabular}

Tabla 4. Conocimientos sobre la realización de la técnica de Diálisis Peritoneal y los balances. 
El apartado 3 estaba destinado a las personas que realizaban D.P. en nuestro servicio (31 enfermeros).

Se les preguntó acerca de las diferentes modalidades de D.P, donde el $80,6 \%(n=25)$ refería conocer la diálisis peritoneal continua ambulatoria (D.P.C.A.) y la diálisis peritoneal automatizada (D.P.A.). Dentro de las diferentes modalidades de D.P.A, el $54,8 \%(n=17)$ conocían la D.P. continua cíclica (D.P.C.C), el 51,6\% $(n=16)$ la D.P. intermitente nocturna (D.P.I.N.), 61,3\% $(n=19)$ la D.P.Tidal y el $19,4 \%(n=6)$ la D.P. continua cíclica plus (D.P.C.C Plus), que son los tratamientos más habituales en nuestro servicio.

El conocimiento de los diferentes líquidos de D.P. es un apartado importante para la realización de la técnica. Los resultados fueron los siguientes: el $12,9 \% \quad(n=4)$ identificó todos los líquidos que utilizan la glucosa como agente osmótico y el 51,6\% ( $n=16)$ identificó el que utiliza los aminoácidos. Cuando preguntamos sobre el tampón utilizado en los líquidos de D.P, el $25,8 \%(n=8)$ identificó correctamente los que utilizan bicarbonato.

El $29 \%(n=9)$ identificó todas las casas comerciales que disponen productos de D.P. y sólo el 58,1\% $(n=18)$ conocía la cicladora que se utiliza en nuestro servicio.

Sobre la pregunta abierta, tuvimos 9 comentarios. Ocho de ellos solicitaban algún tipo de formación en D.P. y uno solicitaba un curso anual de reciclaje para el personal sanitario de nuestro servicio.

\section{Discusión}

La recientemente conversión de nuestro servicio en Unidad de Gestión Clínica ha posibilitado el establecimiento de unos objetivos comunes para el Servicio de Nefrología claramente definidos. Algunos de ellos han sido fijados por recomendación de la Consejería de Salud de nuestra comunidad autónoma, entre los que se encuentra el fomento de la utilización de la D.P. como tratamiento sustitutivo de la función renal.

A pesar de que nuestro hospital fue pionero en el establecimiento de tratamiento domiciliario con D.P. en España, exceptuando el primer periodo de implantación de la técnica ${ }^{8}$, nunca se ha contado con una población importante en el programa de D.P7 . Por lo que las cuestiones planteadas en la introducción, como la posible influencia que puede tener el personal sanitario en la elección del tratamiento, siempre nos las hemos planteado, pero a falta de datos nunca hemos podido resolverlas.

Para conocer el grado de conocimientos sobre la D.P. con el que contaba la Enfermería de nuestro Servicio, pensamos que podía ser útil realizar un cuestionario con preguntas básicas sobre el tratamiento, y a la vista de los resultados, plantear las medidas correctoras más apropiadas, en beneficio de los pacientes así como para completar la formación del personal sanitario.

El personal de Enfermería que respondió $(n=66)$, tenía una amplia experiencia tanto en el ejercicio de la profesión $(20,02+10,16$ años $)$, como en $\mathrm{Ne}$ frología $(15,2+11,1$ años). A pesar de estos datos, sólo el $57,6 \%(n=38)$ había recibido algún tipo de formación en D.P. De estos, el 31,6\% $(n=12)$ no habían realizado ningún tipo de curso de formación con contenido teórico sobre D.P, mientras que el $86,8 \%(n=33)$ habían recibido su formación de forma práctica. Estos datos pueden estar justificados ya que las actividades formativas en D.P. promovidas por el hospital han sido pocas, mientras que sí se ha fomentado y realizado cursos teórico-prácticos de H.D. Sólo acciones fomentadas por el personal de base han sido desarrolladas, incluyendo la organización de varios curso sobre D.P. en los que colaboran y están representados todos los hospitales de nuestra comunidad autónoma con programa de D.P. El número de posibles alumnos de estos cursos autonómicos está restringido por cuestiones presupuestarias, por lo que muchos profesionales interesados en la D.P. no han tenido opción de formarse a través de este medio. En Europa ${ }^{19}$ también se juzga el problema de la cualificación del personal de Enfermería como causa del escaso desarrollo de la D.P.

Acerca de los conocimientos necesarios con los que debe contar Enfermería para realizar el tratamiento en D.P, sólo un $57,6 \%$ de los encuestados sabía realizar un cambio de bolsa y de estos sólo 30,3\% reconocieron manejar adecuadamente una cicladora. El hecho de no existir una proximidad física entre las diferentes Unidades dentro del Servicio, la falta de una práctica habitual de la técnica por un amplio sector del personal ${ }^{20}$, los avances tecnológicos y metodológicos que 
se han producido en los últimos años, junto con una consideración de "sub-especialidad" que puede tener la D.P, pueden justificar estos resultados.

Aunque más del $86,4 \%$ del personal sabían reconocer los síntomas de infección de la inserción del catéter y de la infección peritoneal, un porcentaje superior al 55\% no sabría actuar ante las complicaciones más frecuentes que suelen presentarse en D.P. (hemoperitoneo, infección peritoneal, roturas o desconexión del prolongador, problemas de infusión o drenaje, fuga por la inserción, etc.). Esto conlleva una inseguridad por parte del personal de Enfermería para atender a los pacientes de D.P, situación que los pacientes perciben, desacreditando el tratamiento ante otros pacientes, como ocurrió cuando se implantó el programa, ya que en esa época el sistema de conexión y los medios disponibles no eran los adecuados para prevenir las infecciones peritoneales, creándose una imagen negativa entre pacientes y sanitarios. Con los avances producidos en la actualidad, han disminuido las complicaciones en general, así como la tasa de infecciones peritoneales, pero el mito de la peritonitis continua perviviendo en aquellos colectivos que no han actualizado sus conocimientos.

Sobre las preguntas dirigidas a los profesionales que afirmaron saber realizar el tratamiento con D.P. $(n=31)$, podemos decir que la mayoría $(80,6 \%)$ conocía la D.P.C.A. y la D.P.A. Sin embargo, sólo el 19,4\% conocía la D.P.C.C. Plus. El resto de las diferentes combinaciones de tratamiento que se pueden realizar con D.P.A, fueron identificadas por más del $52 \%$ de los encuestados.

Los diferentes líquidos utilizados en D.P. en función del agente osmótico y del tampón utilizado (glucosa, aminoácidos, icodextrina, bicarbonato, lactato etc.) que se usan para el tratamiento, no fueron debidamente identificados. Esto puede deberse al hecho de que un alto porcentaje del personal que realiza D.P. $(86,8 \%)$, sólo ha recibido formación práctica y con los medios que disponemos en nuestro Servicio, por lo que hay líquidos que no han utilizado. La transmisión oral de esta información quizás no haya sido correcta o mal entendida en algunos casos, lo que justifica ciertos errores en las respuestas sobre temas específicos en este apartado del cuestionario. Este déficit de conocimientos hace que no se pueda informar sobre todas las ventajas que ofrece el tra- tamiento domiciliario con D.P, lo que posiblemente disminuye la probabilidad de que los pacientes opten por esta forma de tratamiento.

Antes los resultados obtenidos, hemos podido comprobar que el grado de conocimiento general sobre la D.P. es limitado. Pensamos que esto puede influir en el fomento y la buena práctica de la D.P. Por otra parte, el $69,2 \%$ de los encuestados piensan que los resultados de esta modalidad de tratamiento para un paciente estándar son iguales o mejores que con la H.D, al igual que ocurre en el trabajo publicado por Ledebo et $\mathrm{al}^{21}$ donde la mayoría de la Enfermería y médicos asistentes a un congreso internacional consideraron que la D.P. era el tratamiento óptimo para iniciar la terapia sustitutiva.

Como conclusión, podemos decir que sería necesaria la corrección de esta situación a través de un curso de contenido teórico-práctico sobre D.P. a través de formación continuada. En la actualidad se está tramitando el mismo para formar y actualizar periódicamente a la Enfermería de nuestro servicio. Pensamos que esta medida favorecería una mejor información a los pacientes con E.R.C.T. sobre las diferentes formas de tratamiento y podríamos comprobar en el tiempo, si repercute en el aumento de los pacientes que optan por la D.P.

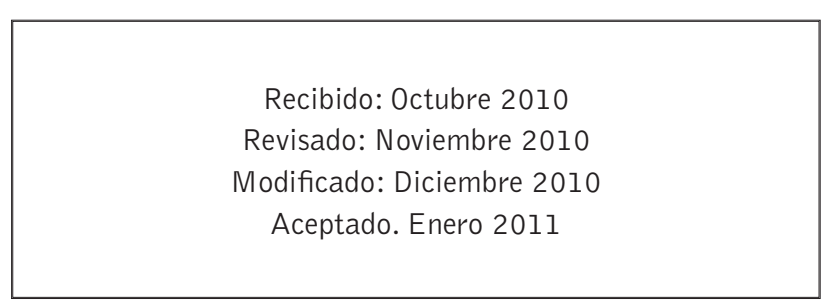

\section{Bibliografía}

1. Korevaar JC, Feith GW, Dekker FW, et al. NECOSAD Study Group. Effect of starting with hemodialysis compared with peritoneal dialysis in patients new on dialysis treatment: a randomized controlled trial. Kidney Int. 2003;64:2222-8.

2. Registro Español de Enfermos Renales. Informe 2006 de diálisis y trasplante renal en España. Nefrología. 2009;29(6):525-533. 
3. Bouvier N, Durand P-Y, Testa A, et al. Regional discrepancies in peritoneal dialysis utilization in France: the role of the nephrologist's opinion about peritoneal dialysis. Nephrol Dial Transplant 2008.

4. Ortega F. Aspectos estructurales en el tratamiento sustitutivo renal. Nefrología. 2010;(1 Suppl Ext 1):21-25.

5. Celadilla 0, Julve M, Vives i Bonjoch A, et al. Evaluación de la información recibida por el paciente que inicia diálisis no programada o procedente de trasplante. En: Libro comunicaciones del XXXII Congreso Nacional de la Sociedad Española de Enfermería Nefrológica; Cádiz 2007. Barcelona: HOSPAL; 2007;38-45.

6. Guías de práctica clínica en Diálisis Peritoneal. Nefrología 2006; 26(Suppl 4).

7. Remón $C$, Quirós $P L$, Gil JM, et al. Diez años de diálisis peritoneal en Andalucía (1999-2008): datos epidemiológicos, tipos de tratamiento, peritonitis, comorbilidad y supervivencia de pacientes y técnicas. Nefrología. 2010;30(1):46-53.

8. Gentil MA, Rivas A, Ramos B, et al. Uso de modalidades de tratamiento de la insuficiencia renal crónica en Andalucía: Influencia de las características del paciente. Nefrología. 2000;(20 Suppl 5):6469.

9. XV Jornada de enfermería de Diálisis Peritoneal. "Percepción de la diálisis peritoneal hoy". Junio 2009. Madrid.

10. Ley 41/2002 de 14 de Noviembre reguladora de la autonomía del paciente y de los derechos y obligaciones en materia de información y documentación clínica.

11. Granado A, Ruiz C, Arrieta, J. Adiestramiento del paciente que se incorpora a la DP. Visitas domiciliarias. Reentrenamiento. Guías de práctica clínicas en DP. Nefrología. 2006;(26 Suppl 4):57-66.
12. Martín JL. Estudio multi-hospitalario sobre diálisis peritoneal ambulatoria continua. En: Libro comunicaciones del XII Congreso Nacional de la Sociedad Española de Enfermería Nefrológica. Vigo 1987. Barcelona: HOSPAL; 1987;195-210.

13. Tejuca Marenco, M. Programa de enseñanza de diálisis peritoneal. En: Libro electrónico de IV Curso Andaluz de DP para Enfermería. Sociedad Española de Enfermería Nefrológica. Madrid 2004;94-98.

14. Gruart, F. Atención de Enfermería en la DP. En: Andreu, L. Forces, E. 500 cuestiones que plantea el cuidado del enfermo renal. Barcelona: Masson, S.A. 1997:220-225.

15. Castro MJ, Sánchez S, Celadilla 0, et al. Enseñanza de las técnicas dialíticas peritoneales. En: Montenegro, J. Olivares, J. (Eds) La diálisis peritoneal. DIBE, S.L. 1999:181-199.

16. Varios autores. Parte II. Enfermería. En: Manual práctico de DP. Coronel F, Montenegro J, Selga R et al. Editorial Atrium, S.L. Badalona. 2005:287-453.

17. Bernardini J, Price V, Figueiredo A, et al. International survey of peritoneal dialysis training programs. Perit Dial Int. 2006;26:658-663.

18. Bernardini, J. Price, V. Figueiredo, A. ISPD Guidelines/Recommendations. Peritoneal dialysis patient training, 2006. Perit Dial Int. 2006;26:625-632.

19. Van Biesen W, Verbeke F, Vanholder R, et al. Why less success of the peritoneal dialysis programmes in Europe? Nephrol Dial Transplant 2008;23:1478-81.

20. Jung $B, B$ lake $P G$, Mehta $R L$, Mendelssohn DC. Attitudes of Canadian nephrologists toward dialysis modality selection. Perit Dial Int 1999;19:263-8.

21. Ledebo I, Ronco C. The best dialysis therapy? Results from an international survey among nephrology professionals. Nephrol Dial Transplant Plus 2008;6:403-8. 


\section{Polyflux}

\section{Para un rendimiento de alto flujo de gran calidad}

- Eliminación eficaz y selectiva de moléculas medianas

- Reducción de efectos inflamatorios y del riesgo de episodios de coagulación debidos a la membrana

- Diseño optimizado

- Los microdominios de la membrana de Polyamix mejoran la biocompatibilidad

- Práctico y fácil de usar 